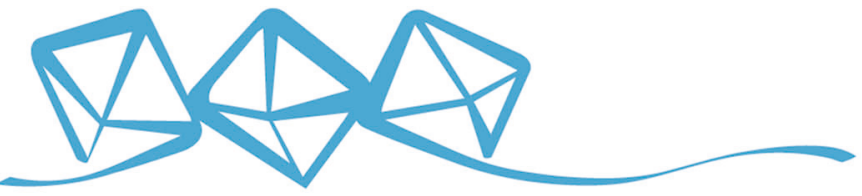 COMMUNICATIONS MATERIALS
}

ARTICLE

https://doi.org/10.1038/s43246-020-00094-y

OPEN

\section{Laser-metal interaction dynamics during additive manufacturing resolved by detection of thermally- induced electron emission}

Philip J. DePond ${ }^{1,2}$, John C. Fuller ${ }^{1,3}$, Saad A. Khairallah¹, Justin R. Angus ${ }^{1}$, Gabe Guss ${ }^{1}$, Manyalibo J. Matthews ${ }^{1}$ \& Aiden A. Martin ${ }^{1 凶}$

In situ monitoring is required to improve the understanding and increase the reliability of additive manufacturing methods such as laser powder bed fusion (LPBF). Current diagnostic methods for LPBF capture optical images, X-ray radiographs, or measure the emission of thermal or acoustic signals from the component. Herein, a methodology based on the thermal emission of electrons - thermionic emission - from the metal surface during LPBF is proposed which can resolve laser-material interaction dynamics. The high sensitivity of thermionic emission to surface temperature and surface morphology is revealed to enable precise determination of the transition between conduction and keyhole mode melting regimes. Increases in thermionic emission are correlated to laser scanning conditions that give rise to pore formation and regions where surface defects are pronounced. The information presented here is a critical step in furthering our understanding and validation of laser-based metal additive manufacturing.

\footnotetext{
${ }^{1}$ Lawrence Livermore National Laboratory, Livermore, CA 94550, USA. ${ }^{2}$ Stanford University, Stanford, CA 94305, USA. ${ }^{3}$ Present address: Colorado School of Mines, Golden, CO 80401, USA. ${ }^{凶}$ email: martin248@IInl.gov
} 
T he market for additive manufacturing technologies has grown exponentially in the past decade and is expected to exceed $\$ 35$ billion in 2024 with more than $40 \%$ growth year-over-year ${ }^{1}$. This growth includes machine manufacturers, component fabricators, and research and development activities by large industry adopters. The quality and reproducibility of additively manufactured components, especially those created for performance critical applications in the aerospace and biomedical industries, is crucial for increased adoption of additive manufacturing ${ }^{2}$. Today's Industry 4.0 paradigm provides increased confidence in fabricated components by creating a so called 'digital thread'; a digital fingerprint of the component that contains life-cycle information such as a computer-aided design schematic, in-process monitoring data, post-processing inspection, and supply chain chronology ${ }^{3,4}$. Laser powder bed fusion (LPBF) additive manufacturing of metals stands to greatly benefit from such enhancements due to the existence of seemingly stochastic events that lead to defects, which limit the performance of fabricated components. Improved monitoring solutions have proven useful in providing some heuristics for defect detection ${ }^{5}$ but many require special integration into the optical path of the processing laser or require additional viewpoints of the powder bed. These solutions are often inaccessible on commercial machines and can be expensive, data intensive or overburdensome to a machine user.

The thermal history of a component fabricated by LPBF additive manufacturing is one of the most important metrics for determining the likelihood of realizing its performance specifications ${ }^{6}$. LPBF fabricates components via a layer-by-layer strategy where a thin layer of metal powder is spread on a solid metal substrate and then selectively melted by a laser. The strategy proceeds by lowering the workpiece and spreading of a new layer of powder, and is repeated until the desired component is formed. The rapid heating and cooling during solidification as well as the numerous thermal cycles during processing introduce unique microstructural features that directly affect part performance. Globally, if the thermal input is too low, the powder layer will not fuse to the underlying component layer and lack of fusion defects can occur. Conversely, if the thermal input is too high, the molten metal evaporates vigorously and a combination of vapor recoil pressure and plasma can form, creating a local depression with increased laser absorption, that is unstable and can lead to porosity. Temperature measurements of the melt pool, which can reach thousands of Kelvin are at the crux of the efforts to describe these phenomena. State of the art in situ temperature measurements rely on thermal imaging 7,8 . These techniques are based on idealized black body thermal emission and are currently the only methods with sufficient temporal and spatial resolutions to capture the thermal dynamics of the process. Thermal gradients on the order of $10^{6} \mathrm{~K} \mathrm{~s}^{-1}$ and meter per second laser scan speed necessitate kilohertz or greater temporal resolution, and laser beam diameters on the order of $100 \mu \mathrm{m}$ dictate spatial resolutions below tens of microns. For LPBF additive manufacturing where the lasermaterial interface is extremely dynamic and consisting of metal vapor, liquid, powder and bulk solid, assumptions that emissivity follow a well-known temperature development are inaccurate. This challenge has limited precise optical temperature measurements to regions with approximately constant emissivity, such as areas behind the melt pool or the cooler solidified regions. Other in situ diagnostic methods used for the probing dynamics during LPBF additive manufacturing include X-ray radiography ${ }^{9-12}$, high-speed optical imaging ${ }^{13,14}$, optical emission spectroscopy ${ }^{15}$, thermal imaging ${ }^{6,16}$, scanning interferometry ${ }^{17}$, and acoustic spectroscopy ${ }^{18,19}$. These methods have proven effective in resolving laser-induced dynamics including melt pool flow, pore formation, surface morphology, vapor plume generation, and powder denudation. By identifying the dynamics that lead to the defects resulting in perturbations in build quality these techniques have improved confidence in LPBF fabricated components.

While rigorous research is being undertaken to understand and measure the process history of an additive manufacturing fabricated component, a thermally dependent signal, thermionic emission, has seemingly been overlooked by the diagnostic community. Thermionic emission detection leverages the phenomena of temperature dependent liberation of electrons, where valence electrons gain sufficient energy to overcome the work function of the material and escape from the metal surface ${ }^{20}$. The thermionic signal has a power-law dependence on the temperature and is described by the Richardson-Dushman equation, $J=A_{G} T^{2} e^{-\frac{W}{k_{B} T}}$, where $J$ is the emission current density, $T$ is the temperature of the metal surface, $W$ is the work function of the material, $k_{B}$ is the Boltzmann constant, and $A_{G}$ is a material specific correction factor ${ }^{20}$. The $W$ of metals are typically greater than $4 \mathrm{eV}^{21}$ resulting in the requirement of very high temperatures, $>2000 \mathrm{~K}$, for appreciable thermionic emission to occur. Therefore, regions of highest temperature in the LPBF processes, i.e., the melt pool region ${ }^{4,22}$, will dominate the measured signal (Fig. 1a, b, and Supplementary Fig. 1). Thermionic emission detection is therefore a promising technique to resolve temperature information during LPBF due to the nonlinear relationship between current density and temperature. While the nature of thermionic emission has been used for signal amplification, electron generation, and electricity generation, to date a sensor of this nature has not been implemented for measurement of metal additive manufacturing processes. The technique does not rely on detection of optical emissions from the surface like other traditional thermal measurement techniques applied to LPBF and is based upon the flow of electrons as they are liberated from the work surface.

In this article, a method to measure the total thermionic signal generated during LPBF is described and used to resolve lasermaterial interaction dynamics in stainless steel $316 \mathrm{~L}$ under LPBF additive manufacturing relevant conditions. Based on the experimental observations thermionic emission is shown to resolve changes in surface temperature caused by changes to the local energy density of the laser beam, surface morphology changes due to transition from conduction to keyhole modes of melting and the formation of a gas discharge at the substrate surface. Correlation of the thermionic signal to surface defects and regions where material overheating leads to pore formation also shows the potential for thermionic emission as an in-process monitoring diagnostic.

\section{Results}

Impact of energy density on the thermionic emission signal. Thermionic emission signals were collected using a custom, testbed LPBF system, which has a high level of control over laser processing conditions ${ }^{23}$ and was modified to include electrical current measurement devices (Fig. 1c). Measurements were conducted such that the thermionic signal, laser parameters and scan location were captured as a function of time so dynamics could be directly correlated to processing conditions and morphological features (Fig. 1d). The pre-build optimization and identification of changes in the beam diameter due to drift and thermal lensing in the focusing optics are critical to maximizing process stability. A study of the thermionic signal as a function of laser beam diameter in stainless steel $316 \mathrm{~L}$ was performed under high vacuum $\left(<10^{-5}\right.$ Torr $)$ and $\sim 750$ Torr of argon environments (Fig. 2). Experiments were performed by varying the distance between the substrate surface and the plano-convex spherical lens used for beam focusing (see Supplementary Fig. 2). At each focus position the corresponding laser beam diameter was used to 
a Surface temperature
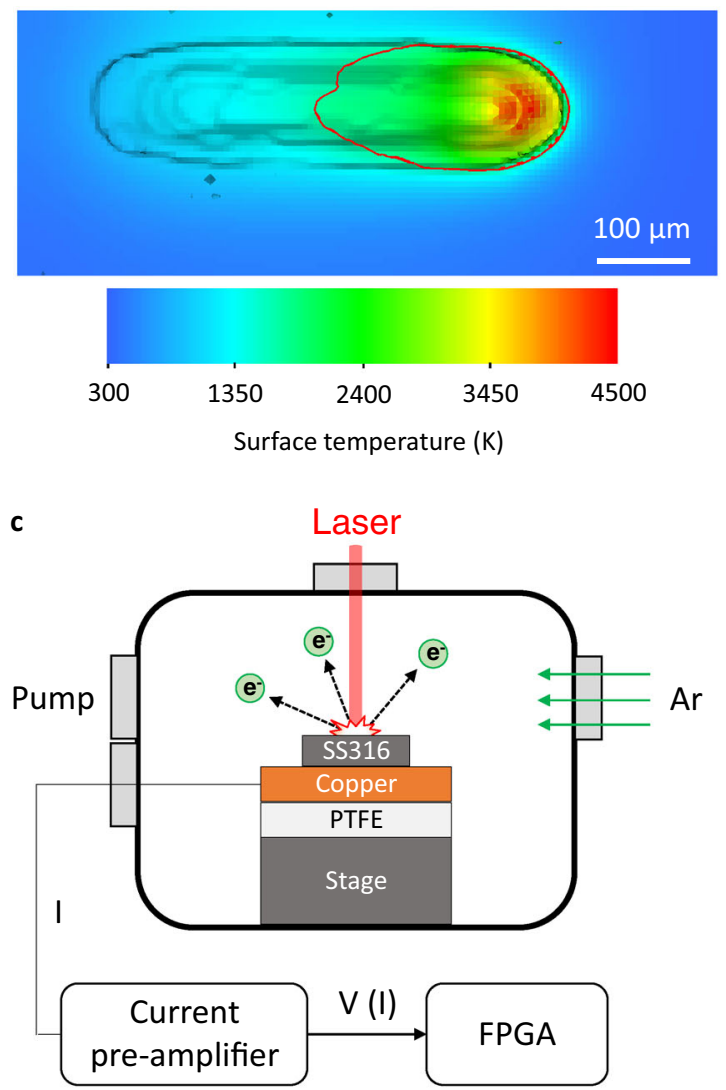

b Thermionic emission
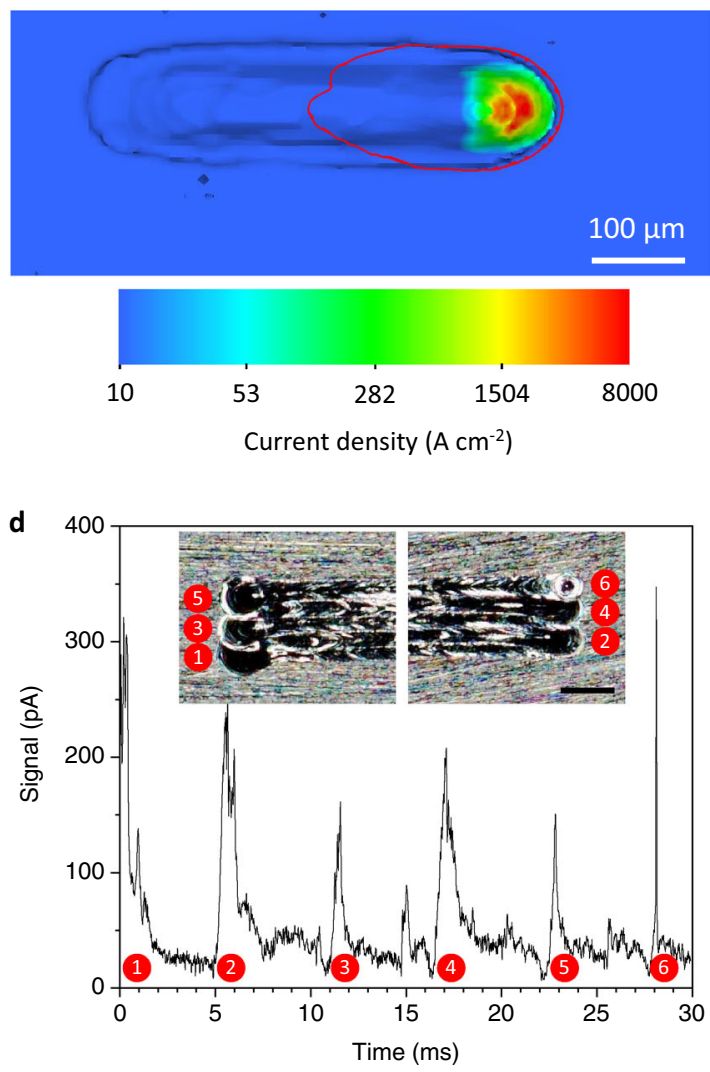

Fig. 1 Visualization of thermionic emission from the substrate surface and the experimental configuration used for signal detection during LPBF. A snapshot from simulations performed using ALE3D of the $\mathbf{a}$ surface temperature and $\mathbf{b}$ thermionic emission profile. The simulations in $\mathbf{a}$ and $\mathbf{b}$ were performed using a stainless steel substrate and laser conditions of $200 \mathrm{~W}$ power, $91 \mu \mathrm{m} \mathrm{D} 4 \sigma$ diameter, and $1500 \mathrm{~mm} \mathrm{~s}^{-1} \mathrm{scan} \mathrm{speed} \mathrm{laser.} \mathrm{The} \mathrm{red} \mathrm{contour}$ line indicates the position of the melt pool boundary. c Simplified schematic of the thermionic emission process initiated by laser irradiation of stainless steel $316 \mathrm{~L}$ and the detection scheme used for signal collection. The flow of electrons during LPBF was measured using a current preamplifier, which was connected to the copper substrate holder. The substrate holder is electrically isolated from the surrounding chamber by a polytetrafluoroethylene (PTFE) block. The current dependent output voltage from the preamplifier is recorded by a field-programmable gate array (FPGA) device. $\mathbf{d}$ Thermionic signal as a function of time collected during irradiation of stainless steel under an argon environment using a $100 \mathrm{~W}$ power, $91 \mu \mathrm{m} \mathrm{D4 \sigma}$ diameter, $1000 \mathrm{~mm} \mathrm{\textrm {s } ^ { - 1 }} \mathrm{scan}$ speed laser and a scan pattern consisting of 5, $5 \mathrm{~mm}$ long lines with $50 \mu \mathrm{m}$ spacing and full power at the turn points. Inset: Optical image of the laser irradiated plate showing the start of track, end of track and turn point regions. The scale bar is $100 \mu \mathrm{m}$.

determine the energy density for calculation of the normalized enthalpy ${ }^{24,25}$, which compares the absorbed laser energy to the amount required to melt the solid. Single laser tracks on bare plate were formed under high vacuum using laser scan conditions of $100 \mathrm{~W}$ power, $1000 \mathrm{~mm} \mathrm{~s}^{-1}$ scan speed and a track length of $10 \mathrm{~mm}$. Tracks in an argon environment were formed using laser scan conditions of $100 \mathrm{~W}$ power, $200 \mathrm{~mm} \mathrm{~s}^{-1}$ scan speed and a track length of $5 \mathrm{~mm}$. These irradiation parameters were chosen as they were found to provide the optimal signal response under the respective environmental conditions. Under high-vacuum conditions a maximum in the thermionic signal of $60 \mu \mathrm{A}$ was realized by performing subsequent scan tracks with incremental steps near and at the focus condition (Fig. 2a). The D4 $\sigma$ diameter of the laser beam at focus was $\sim 90 \mu \mathrm{m}$ and increased to 98 and $108 \mu \mathrm{m}$ at either side of the focus condition respectively. When the mean thermionic signal is compared to the melt pool depth, measured from metallographic cross sections, a direct correlation is observed with the peak thermionic signal corresponding to the maximum melt pool depth. The melt pool depth increases linearly as a function of beam diameter increasing from 14 to $18 \mu \mathrm{m}$, or $29 \%$. Over the same range the thermionic signal increases from $\sim 20$ to $\sim 60 \mu \mathrm{A}$, or $3 \times$ increase. The rapid increase in thermionic signal is ascribed to an increase in the surface temperature of the metal substrate caused by the increase in the local energy density. Asymmetry in the melt pool depth and thermionic signal as a function of beam diameter is observed as the laser steps through the focus condition and is ascribed to the convergent and divergent nature of the laser beam. As the laser steps through the focus condition, the focal plane is located above (divergent) or below (convergent) the surface plane. A convergent beam will produce a deeper melt pool than a divergent beam with the same beam diameter ${ }^{26}$. Figure 2 a shows that both the melt pool depth and thermionic emission signal capture changes in focus condition and beam diameter.

Figure $2 \mathrm{~b}$ shows the mean thermionic signal as a function of laser beam diameter under an argon environment. Like high vacuum, under an argon environment the signal is relatively low at large beam diameters and low normalized enthalpy. As the maximum energy density condition is approached, the signal increases to a maximum of $80 \mathrm{pA}$ and correlated to the maximum melt pool depth. The melt pool depth generally decreases linearly with increasing beam diameter, as has been observed in previous normalized enthalpy studies ${ }^{11}$, but there is an apparent plateau in the melt pool depth for beam diameters of 90 and $150 \mu \mathrm{m}$. The thermionic signal is nominally zero at beam diameters $>150 \mu \mathrm{m}$ and increases superlinearly once the beam diameter approaches 

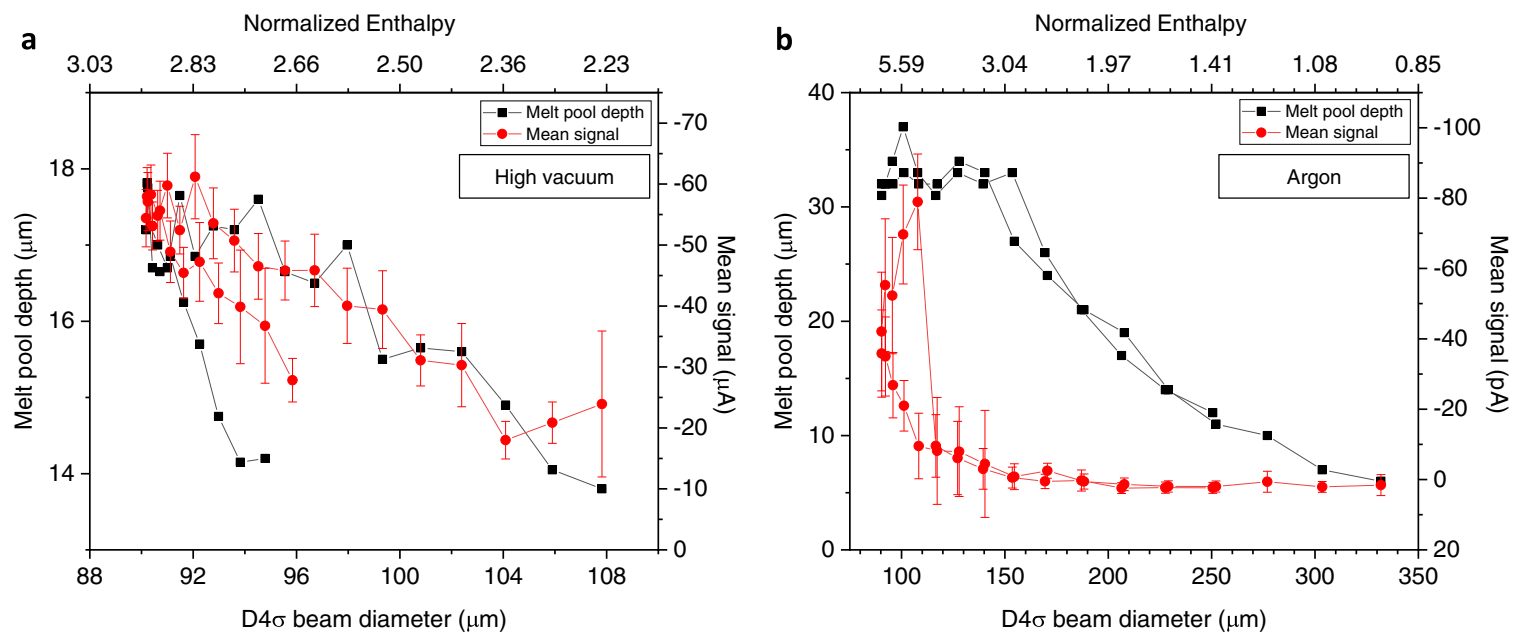

Fig. 2 Mean thermionic signal and melt pool depth as a function of laser beam diameter and normalized enthalpy under varied chamber environments. a Measurements performed in high vacuum. Single $10 \mathrm{~mm}$ long laser tracks in stainless steel were irradiated using a $100 \mathrm{~W}$ power, $1000 \mathrm{~mm} \mathrm{~s}^{-1} \mathrm{scan}$ speed laser. The mean thermionic signal was determined using data collected over a time period of 1-9 ms after the laser turned on. Note the inverted scale of the mean signal. Errors bars indicate standard deviation of the thermionic signal. b Measurements performed in an argon environment. Single $5 \mathrm{~mm}$ long laser tracks in stainless steel were irradiated using a $100 \mathrm{~W}$ power, $200 \mathrm{~mm} \mathrm{~s}^{-1}$ scan speed laser. The mean thermionic signal was determined using data collected over the time period of 1 to $25 \mathrm{~ms}$ after the laser turned on.

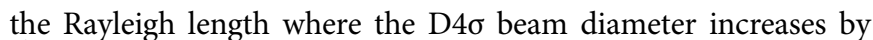
$\sqrt{ } 2, \sim 128 \mu \mathrm{m}$. The magnitude of thermionic signal is dramatically different between high vacuum $(60 \mu \mathrm{A})$ and argon environments $(80 \mathrm{pA})$.

The difference in magnitude of the thermionic signal is attributed to the interaction of emitted electrons with the argon cover gas and subsequent plasma formation above the substrate surface. In high vacuum, electrons are liberated from the substrate and emitted into vacuum away from the substrate unimpeded by gaseous species in the vacuum chamber. In the presence of an ambient gas where the electron mean free path is relatively small $(1-10 \mu \mathrm{m})$, the electrons emitted from the surface will be locally heated by the electric field of the laser. This can lead to strong local amplification of the electron/ion densities and potentially transform the gas near the surface into a plasma discharge. The local ionization rate depends on the local reduced electric field, $E / N$, where $E$ can be estimated using the laser electric field amplitude and $N$ is the local gas density ${ }^{27}$. Assuming the local gas temperature varies between $300 \mathrm{~K}$ and $3000 \mathrm{~K}$, the local gas number density range is $2.45 \times 10^{19}-2.45 \times 10^{18} \mathrm{~cm}^{-3}$. $E$ was calculated as $4.81 \times 10^{4} \mathrm{~V} \mathrm{~cm}^{-1}$ assuming a laser power of $200 \mathrm{~W}$ and a $\mathrm{D} 4 \sigma$ beam diameter of $91 \mu \mathrm{m}$. This gives $E / N$ values in the range of $200-2000 \mathrm{Td}$, where $\mathrm{Td}=10^{-17} \mathrm{~V} \mathrm{~cm}^{-2}$ and local ionization rates $\left(v_{i}\right)$ exceeding $10^{10} \mathrm{~Hz}$ assuming an electron energy of $7 \mathrm{eV}^{27}$. The local dwell time $\left(t_{\mathrm{d}}\right)$ of the laser is approximately $500 \mu \mathrm{s}$, where $t_{\mathrm{d}}=\frac{D 4 \sigma}{u}$ and $u$ is the laser scan speed $^{25,26}$. Thus, the exponential amplification factor, $\operatorname{Exp}\left(v_{\mathrm{i}} t_{\mathrm{d}}\right)$, of an electron is $\operatorname{Exp}\left(5 \times 10^{6}\right)$, and rapid formation of a plasma is expected. The presence of a plasma above the surface in the argon experiments can explain the large, six orders of magnitude decrease in the measured current. Since no voltage is applied, the plasma will be driven towards its natural floating potential with respect to the metal substrate such that the net flux of ions and electrons to the surface is balanced and there is no net current ${ }^{28}$, effectively shielding electrons from escaping the metal surface. The formation of a plasma sheath at the plasma-substrate interface could also potentially explain the polarity switch and timedependent behavior of the current traces in an argon gas. This hypothesis requires further examination and a detailed investigation is beyond the scope of this article. It is important to note that at reduced pressures, the Clausius-Clapeyron equation shows a reduced vaporization temperature as compared with typical atmospheric pressure conditions typically seen in $\mathrm{LPBF}^{29,30}$. The recoil pressure, the difference between the pressure exerted by the laser plume on the melt pool and the ambient pressure, is also reduced at atmospheric pressure ${ }^{13,29,30}$. Differences in recoil pressure and ambient pressure under these two pressure regimes (high vacuum and argon atmosphere) will affect the melt pool morphology and surface temperature, and therefore the thermionic signal. The changes induced by these mechanisms, however, is not expected to be significant enough to explain the six orders of magnitude difference in the measured signal.

Thermionic signal as a function of laser power and scan speed. To understand the dependence of laser parameters on the thermionic signal, various laser power and speed combinations were investigated in stainless steel $316 \mathrm{~L}$ bare plates under high vacuum and argon environments (Fig. 3). The range of laser parameters used are typical for LPBF of stainless steel $316 \mathrm{~L}^{31,32}$. In high vacuum, the polarity of the measured thermionic signal is negative meaning electrons are being emitted from the metal surface. The thermionic signal increases in magnitude with increasing laser power. At slower scan speed, $200 \mathrm{~mm} \mathrm{~s}^{-1}$, the signal at $50 \mathrm{~W}$ is minimal, $<20 \mu \mathrm{A}$, and when the laser power increased to $150 \mathrm{~W}$ the signal magnitude increases greatly, approaching $100 \mu \mathrm{A}$ (Fig. 3a). At $150 \mathrm{~W}$ the signal noise also increases significantly compared to those at lower laser powers. When the power is increased above $150 \mathrm{~W}$ the magnitude of the signal does not increase, however, while the noise of the signal increases significantly. The cause of this increase in noise is ascribed to transition from conduction to deep keyhole mode melting 9 and is discussed at length in the next results section. The signal at the start and end of track is greater than the steady state scanning condition and is caused by a decrease in the scan speed during these phases of the track due acceleration and deceleration of the galvanometer scanning mirrors ${ }^{11}$. Measurements performed at $1000 \mathrm{~mm} \mathrm{~s}^{-1}$ scan speed show an almost linear increase in the magnitude of the thermionic signal with laser power until $300 \mathrm{~W}$ where the signal magnitude plateaus (Fig. $3 \mathrm{~b}$ and Supplementary Fig. 3). Inspection of optical images of cross sections under these 
a Vacuum, $200 \mathrm{~mm} \mathrm{~s}^{-1}$

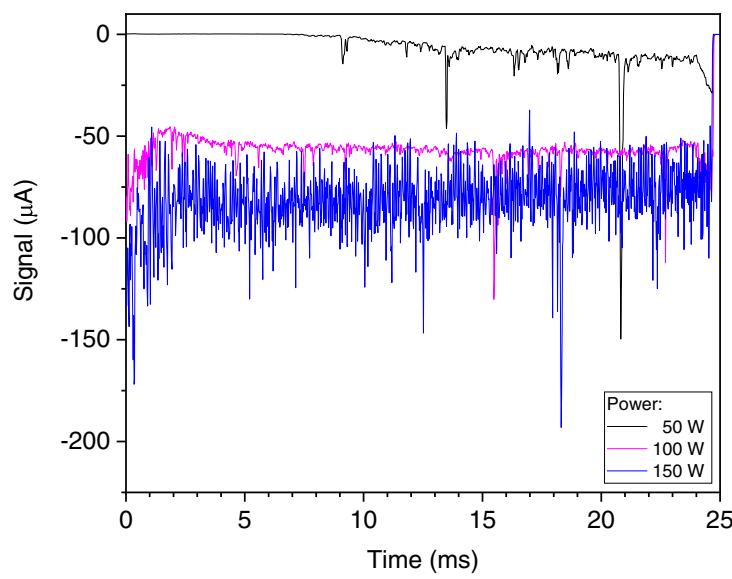

c Argon, $200 \mathrm{~mm} \mathrm{~s}^{-1}$

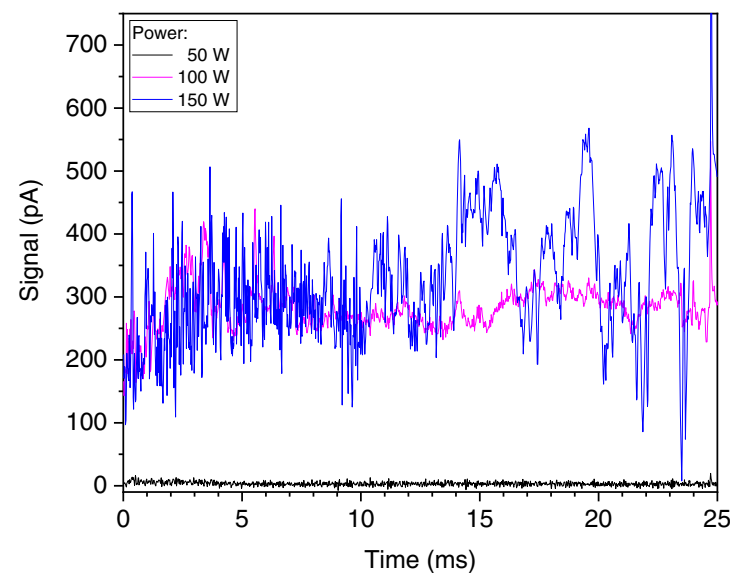

b Vacuum, $1000 \mathrm{~mm} \mathrm{~s}^{-1}$

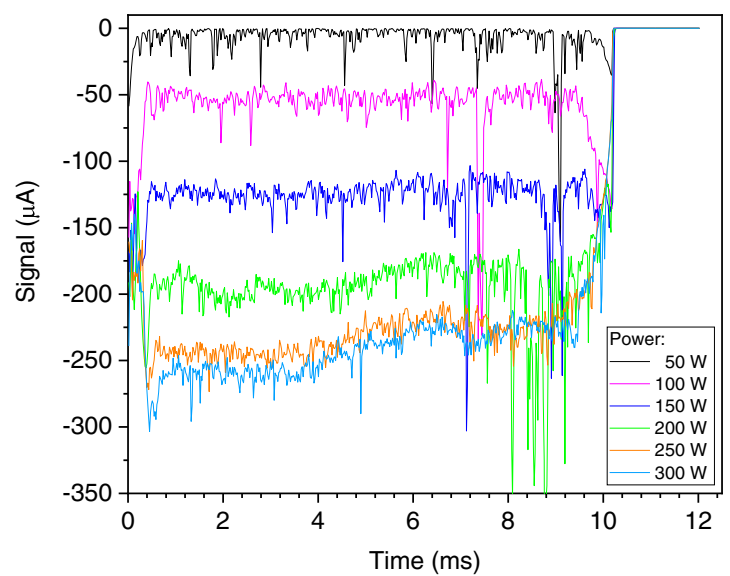

d Argon, $1000 \mathrm{~mm} \mathrm{~s}^{-1}$

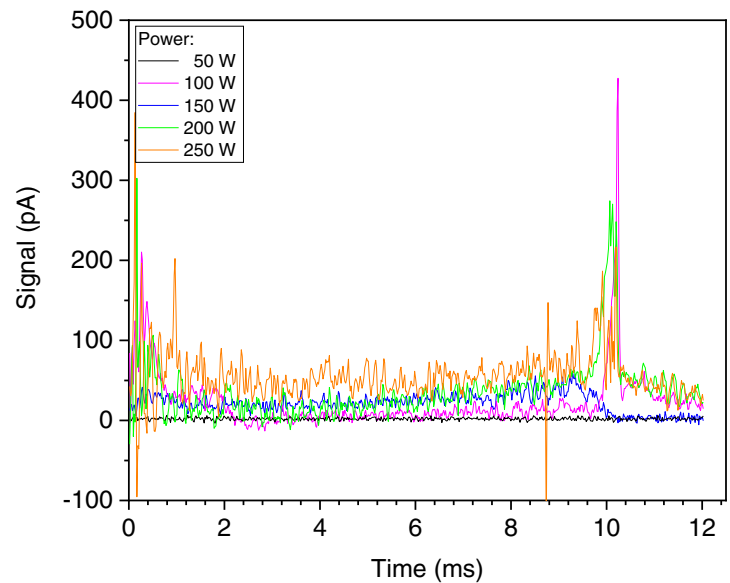

Fig. 3 Thermionic signal as a function of time under various chamber environments and laser scan conditions. $\mathbf{a}$, $\mathbf{b}$ Signal as a function of time for single $10 \mathrm{~mm}$ long tracks in stainless steel performed under high vacuum as a function of laser power using a laser scan speed of $\mathbf{a} 200$ and $\mathbf{b} 1000 \mathrm{~mm} \mathrm{~s}^{-1}$. c, $\mathbf{d}$ Signal as a function of time for single $5 \mathrm{~mm}$ long tracks in stainless steel performed under an argon environment as a function of laser power using a laser scan speed of $\mathbf{c} 200$ and $\mathbf{d} 1000 \mathrm{~mm} \mathrm{~s}^{-1}$.

laser power and scan speeds reveal that the $300 \mathrm{~W}$ case is at the onset of the keyhole mode melting transition and these transition dynamics are ascribed to the observed change in the linear response of the thermionic signal with laser power (see Supplementary Fig. 3).

Laser irradiation under an argon environment results in a thermionic signal with positive polarity (Fig. 3c). The positive signal indicates that electrons are being absorbed by the metal during laser heating under an argon environment. The flow of electrons into the metal surface was unexpected and is ascribed to a non-uniform distribution of free electrons and ions in a plasma $^{33}$. For measurements performed at $200 \mathrm{~mm} \mathrm{~s}^{-1}$ scan speed, the argon results are similar to the high-vacuum case with the irradiation performed at $50 \mathrm{~W}$ laser power generating minimal thermionic signal. Note, however, the six orders of magnitude difference in thermionic signal between the two environmental conditions under identical laser parameters. As the laser power increases the signal increases in magnitude to $\sim 500 \mathrm{pA}$. At higher laser power, the signal also possesses a plateau and increase in noise. When the scan speed is increased to $1000 \mathrm{~mm} \mathrm{~s}^{-1}$ significant changes in thermionic signal are only observed at the start and end of the track (Fig. 3d). Even at $300 \mathrm{~W}$ the signal during steady state scanning is only on the order of 50 $\mathrm{pA}$. For the case of argon at high scan speeds the thermionic signal therefore appears less responsive to changes in laser power and more so to changes in scan speed. The parameter dependence results show that the thermionic signal is responsive to changes in-process parameters especially in vacuum where electrons can freely escape the surface and the signal scales laser power until a threshold is reached at the transition to keyhole mode melting. The thermionic signal response in argon is more nuanced and is greatly affected by the plasma formed in the argon shielding gas.

Thermionic signal response to keyhole transition. The increase in noise of the thermionic signal with increasing laser power was investigated using fast Fourier transform (FFT) analysis of the dataset collected under an argon environment using $200 \mathrm{~mm} \mathrm{~s}^{-1}$ laser scan speed processing conditions (Fig. 4a, b). Figure $4 \mathrm{a}$ shows the thermionic signal as a function of time for $5 \mathrm{~mm}$ long tracks on bare plate produced at laser powers from 50 to $300 \mathrm{~W}$. The noise in the thermionic signal was quantified using FFT analysis to calculate the power as mean squared amplitude (MSA) at lower frequencies, $<100 \mathrm{kHz}$, in the Fourier domain of data collected from the entire length of each respective track (Fig. 4b). As shown in the inset of Fig. $4 \mathrm{~b}$ the power as MSA at $15 \mathrm{kHz}$ increases sharply at laser powers $>150 \mathrm{~W}$. The melt pool morphology of each respective track was examined by cross sectioning and optical imaging. The melt pool for the track produced at $50 \mathrm{~W}$ is very shallow and the width and depth of the melt pools 


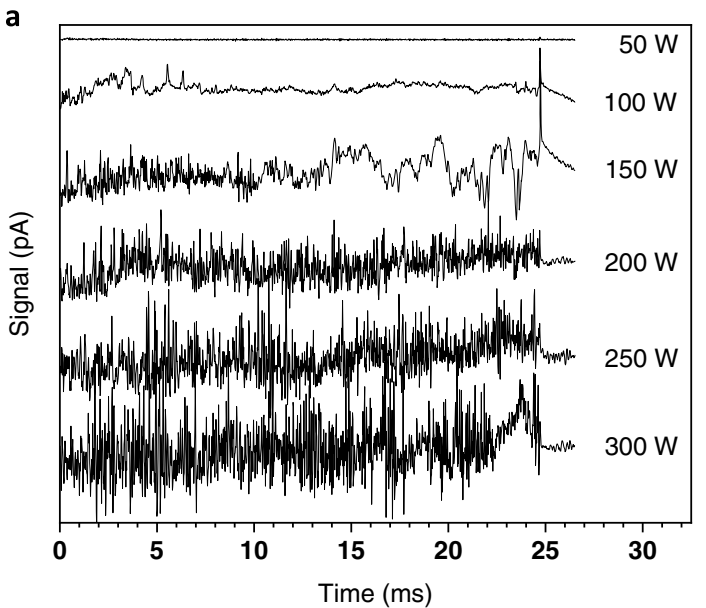

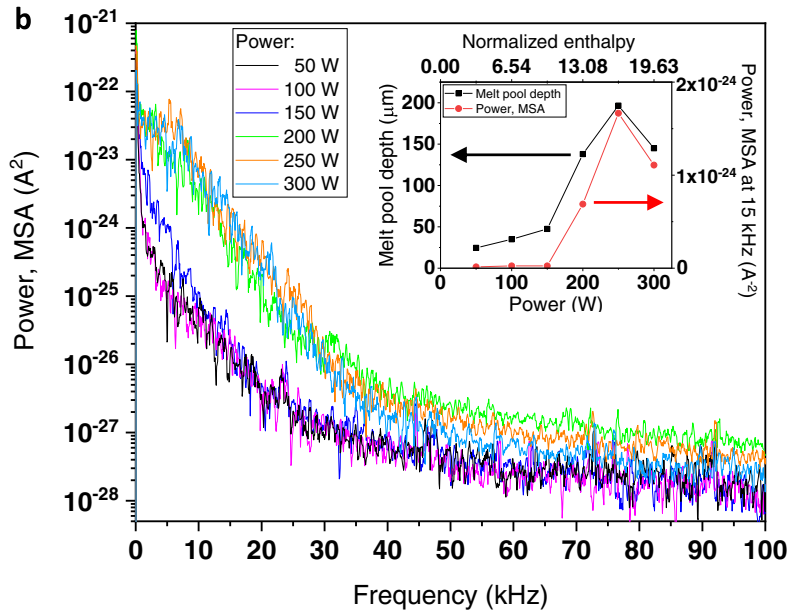
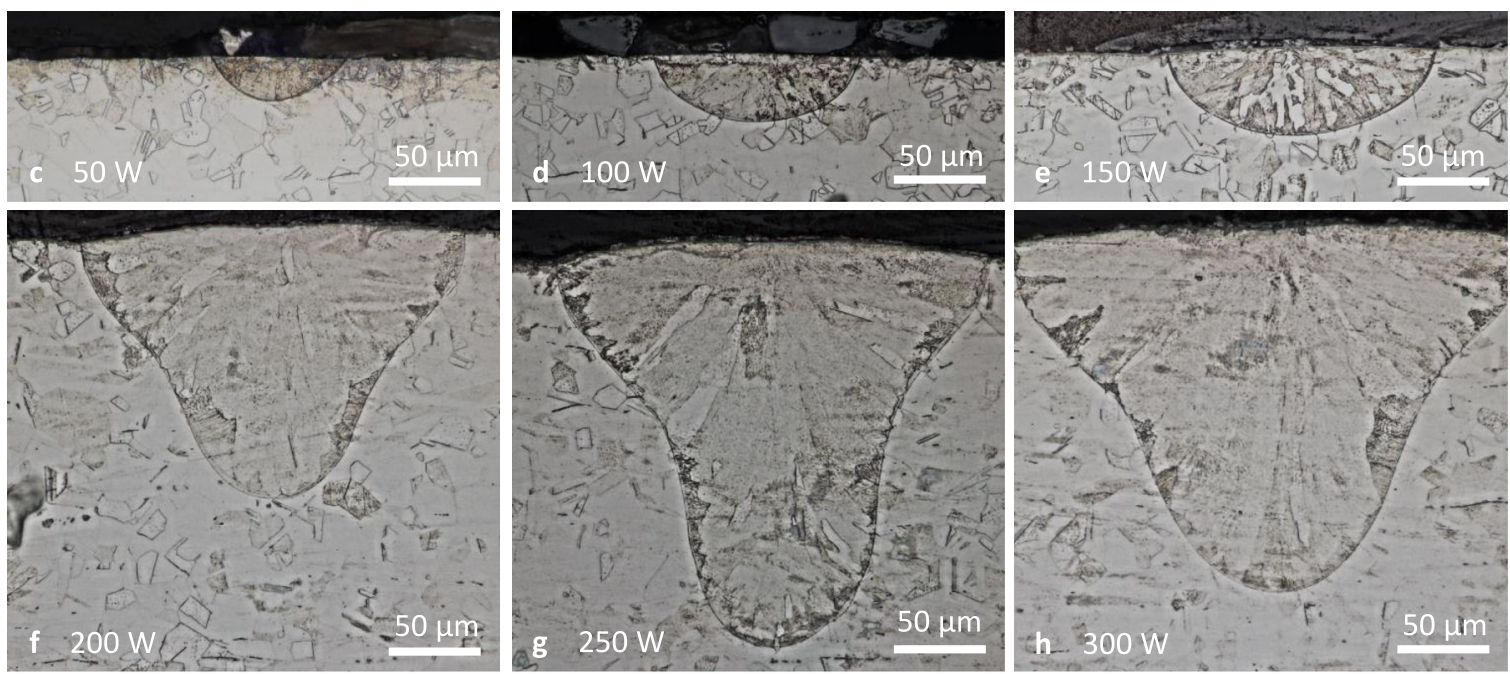

Fig. 4 Identifying the transition to keyhole mode melting using FFT analysis of the thermionic signal. a Thermionic signal as a function of time for single $5 \mathrm{~mm}$ long tracks in stainless steel performed under an argon environment as a function of laser power using a laser scan speed of $200 \mathrm{~mm} \mathrm{~s}{ }^{-1}$. $\mathbf{b}$ FFT of the thermionic signals shown in a displayed in the form of power as a function of frequency. Inset: Melt pool depth and FFT power at $15 \mathrm{kHz}$ as a function of laser power. c-h Optical images of cross sections of tracks produced in a revealing melt pool geometry as function of laser power.

increases at 100 and $150 \mathrm{~W}$ laser power (Fig. 4c-e). These melt pools display regular elliptical shapes and are in the conduction mode of laser melting. For the tracks produced at 200, 250, and $300 \mathrm{~W}$ the cross sections show the welds are in the keyhole mode melting regime, possessing the characteristic chalice shape (Fig. 4f-h). Comparing the depth of the melt pool as a function of laser power and the FFT analysis, an abrupt increase in melt pool depth occurs at the same laser power threshold as the increase observed in power as MSA at $15 \mathrm{kHz}$ (Fig. $4 \mathrm{~b}$ ). The increase in power as MSA at $15 \mathrm{kHz}$ is therefore attributed to the transition from conduction mode to keyhole mode melting. This trend is similar to that observed during in situ measurement of laser energy absorption where the absorptivity and melt pool depth sharply increases during the transition to keyhole mode melting $^{34}$. The frequency response indicating keyhole mode instabilities also matches previous observations using in situ X-ray imaging ${ }^{10}$. The data reveals a direct correlation between the transition to keyhole mode melting and the increase in thermionic signal noise, providing a pathway for readily identifying the transition in real-time during laser irradiation. The onset of keyhole depression formation also corresponds to a plateau in the measured thermionic signal, which could be explained by limiting of the surface temperature due to boiling of the metal or reduced efficiency for electrons escaping the substrate due to the surface morphology. Note that space charge effects ${ }^{35}$ could also play a role in the plateau; however, this cannot easily be predicted due to the tangential nature of the electric field produced by the laser. Until recently, when vapor depression dynamics were probed using in situ X-ray imaging 9 , the characterization of conditions prone to the formation of keyhole morphology depression were based on indirect cross-sectional measurements. Keyhole depressions form under high energy density conditions where a large recoil pressure is generated resulting in the drilling of a deep depression in the melted metal, forming a high-aspect-ratio metal vapor filled cavity. During irradiation, the processing laser interacts with the vapor depression's surface where light is absorbed or reflected generating localized perturbations within the depression, which can lead to pore formation due to entrapment of gas bubbles in the melt pool ${ }^{10,22,24}$ and oscillations in the surface morphology ${ }^{10}$. Identifying keyhole mode melting is therefore important for mitigating pore formation, which degrades the fatigue life of LPBF fabricated components by acting as crack initiators ${ }^{36}$.

Thermionic signal response during LPBF scan strategies. Conditions replicating the LPBF additive manufacturing process 
a Thermionic signal

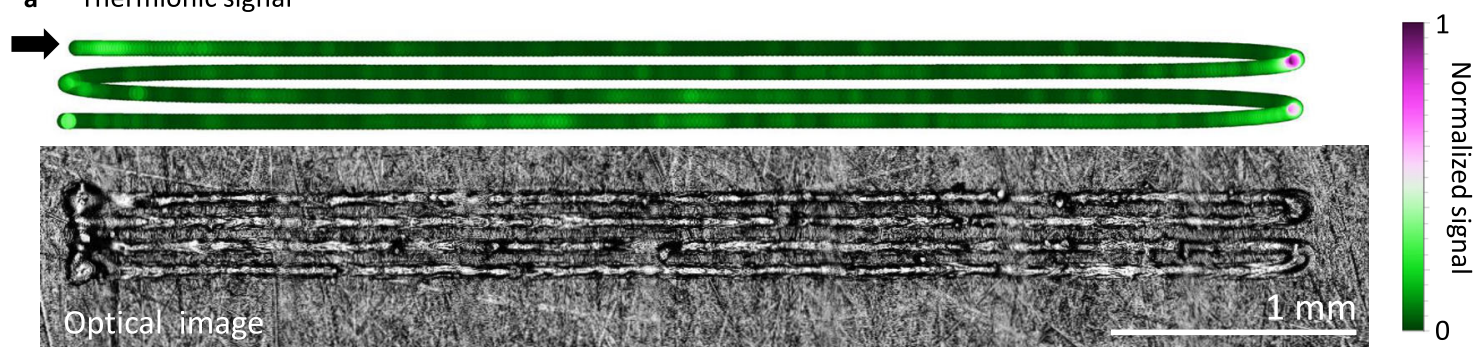

b

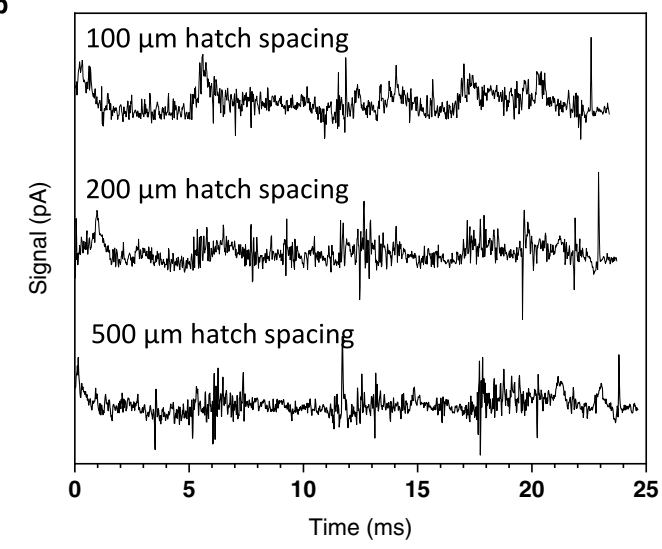

d

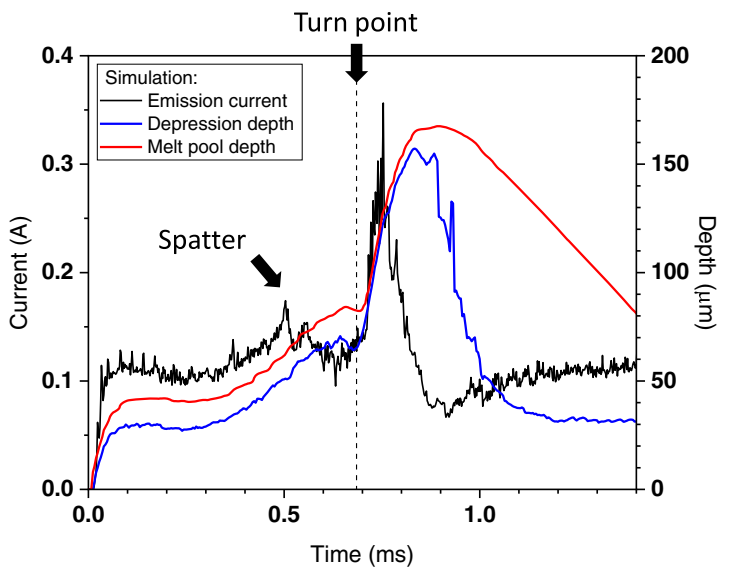

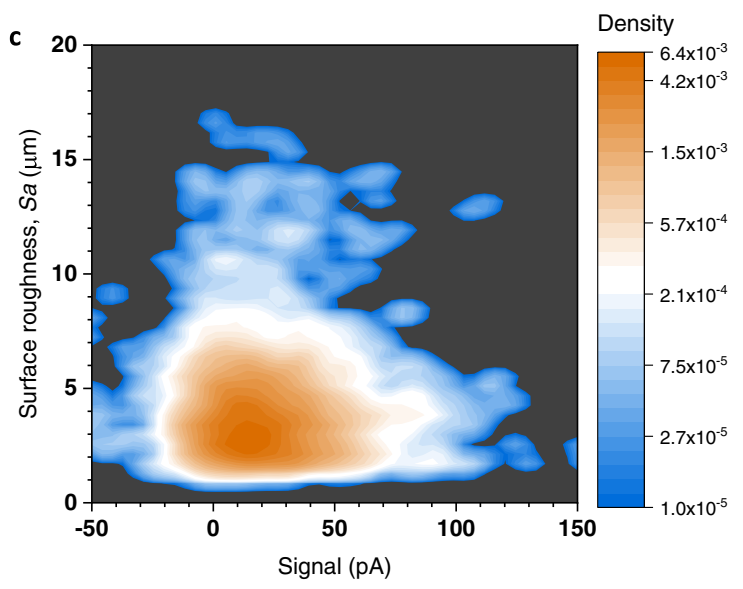

e
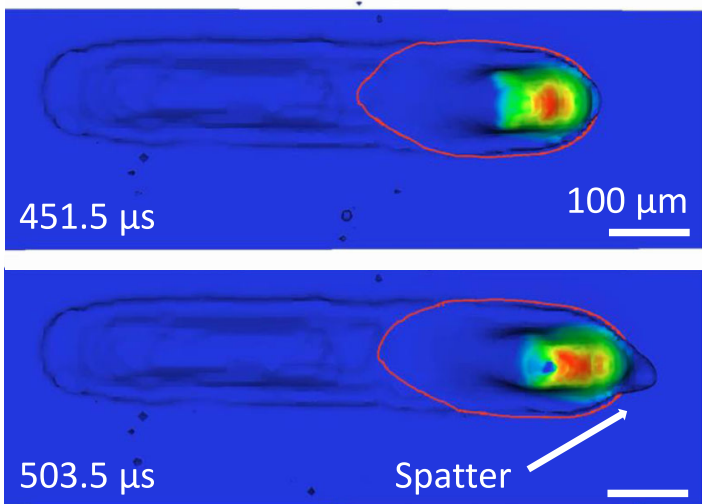

Fig. 5 Measured and simulated thermionic signal response under LPBF-like, hatch scan strategy conditions. a Thermionic signal intensity as a function of scan position in stainless steel $316 \mathrm{~L}$ performed under an argon environment using $100 \mathrm{~W}, 1000 \mathrm{~mm} \mathrm{~s}^{-1}$ laser parameters and $4,5 \mathrm{~mm}$ long scan lines with $100 \mu \mathrm{m}$ hatch spacing. The arrow indicates the start of track position. Also shown is an optical image of the formed tracks. $\mathbf{b}$ Thermionic signal as a function of time under the same laser conditions used in a with 100,200, and $500 \mu \mathrm{m}$ hatch spacing. c A two-dimensional kernel density plot consisting of the thermionic signal measured from tracks shown in $\mathbf{b}$ during steady state scanning conditions and the local surface roughness determined from height data collected ex situ using a laser scanning confocal microscope. Note the logarithmic color scale. $\mathbf{d}$ Electron current emitted from the metal surface, and vapor depression and melt pool depth as a function of time during a single laser turn point simulated using an ALE3D based multi-physics model. The steady state scan speed was $1500 \mathrm{~mm} \mathrm{~s}^{-1}$ and reduced to $750 \mathrm{~mm} \mathrm{~s}^{-1}$ during the turn. A constant laser power of $200 \mathrm{~W}$, a hatch spacing of $100 \mu \mathrm{m}$ and a D4 $\sigma$ beam diameter of $91 \mu \mathrm{m}$ were used in the simulation. e Surface profiles captured during the simulation in $\mathbf{d}$ at 451.5 and $503.5 \mu$ s. The image is colorized using a logarithmic scaling of the electron current density. The red contour line indicates the position of the melt pool boundary.

were investigated using a thin layer of powder on the substrate surface and hatched scanning patterns (Fig. 5). A series of bidirectional hatches were produced on stainless steel $316 \mathrm{~L}$ with varied hatch spacing. These hatches mimic a unit of the 'island' or 'chess' scan strategies in commercial LPBF systems. Three $5 \mathrm{~mm}$ islands with 100,200 , and $500 \mu \mathrm{m}$ hatch spacing were scanned using a laser power of $100 \mathrm{~W}$ and scan speed of $1000 \mathrm{~mm} \mathrm{~s}^{-1}$ to observe the effect of turn point velocity and overheating on the measured thermionic signal (Fig. 5a, b). With a hatch spacing of $100 \mu \mathrm{m}$ the thermionic signal peaks at the start of track and laser turn points, and multiple spikes in the signal are observed during steady state scanning (Fig. 5a). The signal intensity at turn point regions increases with decreasing hatch spacing. This is caused by the laser scanning mirrors requiring a slower scan velocity at laser turn points, which varies depending on the scan profile (see Supplementary Fig. 4). For example, the turn point velocity approaches a minimum of $\sim 50 \mathrm{~mm} \mathrm{~s}^{-1}$ for the $100 \mathrm{um}$ hatch spacing irradiation. The change in velocity causes the process laser to dwell at these points for substantially longer than in the steady state scan regime resulting in an increase in the local 
energy density which causes overheating and unstable keyhole mode melting ${ }^{11}$. Interestingly, the thermionic signal is at maximum intensity when the laser reaches the turn point and then begins to decrease when the laser accelerates out of the turn point (Fig. 5a). This signal change occurs during localized melt pool transitions between conduction and keyhole mode melting and is a major source of pore defects during $\mathrm{LPBF}^{11}$. While the maximum thermionic signal is apparent at the turn point regions, local maxima along the steady state scanning regions appear to correlate with surface inhomogeneities. These surface defects are undesirable and can result in build defects such as porosity from lack of fusion. To quantify this observation, analysis of the local surface roughness $(S a)$ and thermionic signal was performed using a two-dimensional kernel density plot to estimate the probability distribution function (Fig. 5c). The plot shows that the highest density of data points has a thermionic signal magnitude on the order of $\sim 15 \mathrm{pA}$ and a surface roughness of $\sim 2.5 \mu \mathrm{m}$. Regions with a thermionic signal up to $\sim 50 \mathrm{pA}$ are much more likely to possess a higher surface roughness than those $>50 \mathrm{pA}$. As the signal increases the surface roughness is generally smoother, which likely arises from overheating and slower cooling of the surface.

High-fidelity multi-physics simulations were used to identify key relationships between thermionic emission and melt pool dynamics during a laser turn point (Fig. 5d and see Supplementary Movies 1-3). The simulation was performed at $200 \mathrm{~W}$ laser power, a steady state scan speed of $1500 \mathrm{~mm} \mathrm{~s}^{-1}$, a turn point speed of $750 \mathrm{~mm} \mathrm{~s}^{-1}$, and a hatch spacing of $100 \mu \mathrm{m}$. Only relative changes were compared between experiment and simulation due to the uncertainty in the value of the material specific constants, $A_{G}$ and $W$, with temperature ${ }^{21}$ and the physical state of the material ${ }^{37}$. The simulated emission current is approximately $10^{3}$ orders of magnitude larger during steady state scanning compared to experiment, $100 \mathrm{~mA}$ versus $100 \mu \mathrm{A}$. The experimental collection efficiency of emitted electrons from the surface could also contribute to the difference in signal. An increase in thermionic emission current is observed when the laser reaches the turn point, reaches a maximum $\sim 75 \mu$ s later and then decreases in $\sim 150 \mu$ s as the laser accelerates out of the turn. This response and the asymmetric profile of the thermionic signal closely matches the experimental observations. When the melt pool response is compared to the thermionic emission signal it can be noted that the maximum thermionic signal precedes the maximum melt pool and depression depth by $\sim 150 \mu$ s. This is because the response of the melt flow to the local maximum in recoil pressure is delayed by the liquid's inertia, but electron emission responds almost instantaneously relative to the temperature increase. The simulations also reveal another change in melt pool morphology which correlates to changes in the thermionic signal. A local maximum in the thermionic signal at $\sim 500 \mu$ s corresponds to the formation of a liquid droplet ejecta (Fig. 5e), which is also a potential source of defects during LPBF additive manufacturing ${ }^{38}$. As the ejecta forms, the melt pool elongates into a bow wave-like shape before ejection ${ }^{39}$, increasing the surface area of the highest temperature region at the front wall where the laser couples to the surface. The results from experiments replicating LPBF additive manufacturing and accompanying multi-physics simulations show that overheating and changes in melt pool morphology during in laser irradiation, such as at laser turn points and during liquid droplet ejection can be readily identified via detection of the thermionic emission signal. The observation of thermal changes at the substrate surface with a method such as thermionic emission detection using electron current measurement devices could lead to realtime methods for defect identification and ultimately increased confidence fabricated components.

\section{Discussion}

In summary, we have uncovered the generation of thermionic emission during LPBF additive manufacturing and used the signal to identify dynamics caused by laser-metal interactions. Thermionic emission was resolved in a common LPBF additive manufacturing material, stainless steel $316 \mathrm{~L}$, under various laser scanning conditions by measuring the flow of electrons between the metal surface and the local chamber environment using a current preamplifier. Variation of the laser beam diameter shows that the thermionic emission signal exponentially increases with the local energy density, and the melt pool depth increases linearly over the same range. These relationships reveal the critical dependence of surface temperature on the thermionic emission signal and the applicability for using the signal as a methodology for rapid optimization of laser focus in LPBF additive manufacturing systems. The thermionic signal increases linearly with laser power under high-vacuum conditions until the keyhole mode melting regime is realized. The signal response under argon at high laser scan speeds is more dependent on changes in surface morphology and signal maxima are caused by changes in laser scan speed at the start and end of track. The measurement of a positive current polarity in argon and the six orders of magnitude difference between high vacuum and argon environments was entirely unexpected. These observations point to the role of plasma formation being a key phenomenon associated with the measured signal. The positive polarity is caused by electrons flowing from the plasma plume into the metal substrate. Our current hypothesis is that this is related to the dynamic formation of a plasma sheath at the plasma-substrate interface. The observation of thermionic emission reveals that the formation of plasma during LPBF additive manufacturing previously ascribed to ionization of vaporized metal by the laser beam, could also be caused by liberation of electrons from the surface of the metal into the argon cover gas and subsequent interaction with the large electric field of the laser. Analysis of the thermionic signal using FFT can identify the transition of laser melting dynamics from conductive to keyhole mode melting by observing a sharp rise in the noise component at frequencies $<30 \mathrm{kHz}$. The identification of this transition is desirable as keyhole formation results in pore formation due to its unstable nature and importantly, unlike other in situ techniques such as $\mathrm{X}$-ray imaging, thermionic emission can resolve this transition in bulk material. Locations of overheating due to poorly configured processing conditions and stochastically formed surface defects were identified during hatched scan patterning of a powder covered substrate using the thermionic signal for in-process monitoring. State of the art multi-physics simulations corroborate the relationship between increased melt depression depth and thermionic signal including perturbations in the melt pool that lead to material ejection (i.e., spatter) and increased signal. The simulations also reveal fundamental differences in the hydrodynamic and electrical response to changes in surface temperature, which is ascribed to the time required for the increased recoil pressure to overcome the melt pool's inertia. The results demonstrate that the collected thermionic signals can be incorporated into conventional data collection schemes and processing methods including FFT and signal threshold analysis. Understanding the response of diagnostic signals to lasermaterial interactions during LPBF processing and identifying regimes where defects may arise is a key step for developing confidence in additively manufactured components. The results presented here illustrate the potential for thermionic emission detection to provide detection of laser driven phenomena and improve our knowledge of the LPBF additive manufacturing process whilst also complimenting existing diagnostic capabilities. 


\section{Methods}

Experimental design. Laser-induced thermionic emission signals were collected for a series of single laser tracks and hatches on stainless steel $316 \mathrm{~L}$ substrates as a function of chamber pressure, laser beam diameter, laser power and laser scan speed. 6.35-mm thick stainless steel 316 L substrates (SS316L, Rolled Alloys Inc., see Supplementary Table 1 for material composition details) were investigated with and without a thin layer of stainless steel $316 \mathrm{~L}$ powder $(50-65 \mu \mathrm{m}$ layer thickness, 15-45 $\mu \mathrm{m}$ diameter powder size distribution, Concept Laser) on the surface. Experiments performed under an $\sim 750$ Torr argon environment were conducted by flowing ultra-high purity argon gas via a mass flow controller (Smart-Trak 100, Sierra Instruments) at $\sim 0.7$ standard liters per minute while actively evacuating the chamber with a diaphragm pump. Prior to flowing argon, the chamber was evacuated to $<10^{-3}$ Torr to ensure removal of oxygen and nitrogen from the chamber. High-vacuum experimental conditions $\left(<10^{-5}\right.$ Torr $)$ were realized using a turbo molecular pump based system (HiCube80, Pfeiffer Vacuum). The chamber pressure was determined using a gas species-independent pressure transducer $(910$ DualTrans, MKS Instruments). The concentration of oxygen in the chamber was monitored by an oxygen vacuum probe (XS22, ZIROX Sensoren und Elektronik $\mathrm{GmbH}$ ) and was $<10$ parts per million (ppm) for all experiments. Reducing the oxygen concentration is essential as oxygen alters the melt pool geometry during irradiation $^{30}$, changes the gaseous composition of the plasma and can result in formation of metal oxides that tend to have higher work functions than their elemental metals ${ }^{40}$. At near atmospheric concentrations of oxygen $(100,000 \mathrm{ppm})$ the thermionic signal was found to be erratic with large deviations from the nominal average. The magnitude of the thermionic signal as a function of oxygen concentration was minimum at concentrations $<\sim 10 \mathrm{ppm}$, therefore this upper threshold was used for all experiments (see Supplementary Fig. 5).

Experiments were performed using a custom built, testbed LPBF system ${ }^{23}$. This customizable, vacuum chamber-based system was built to mimic typical laser scanning conditions found in a commercial LPBF platform while including a flexible laser optical pathway and advanced diagnostic instrumentation. A singlemode, $1070 \mathrm{~nm}, 500 \mathrm{~W}$, continuous wave fiber laser (YLR-500-WC, IPG Photonics) was used in all experiments as the primary energy source. A modified 3axis galvanometer scanning mirror system (3XB Scan Head, Nutfield Technology) was used to scan the laser across the substrate. The laser focusing optics consisted of an $85 \mathrm{~mm}$ focal length collimator (D25-F85 AC, IPG Photonics) and a $600 \mathrm{~mm}$

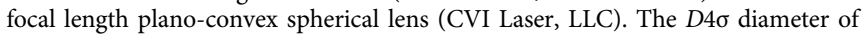
the Gaussian beam at the sample plane was $\sim 90 \mu \mathrm{m}$ (see Supplementary Fig. 2) which was determined using a custom beam profiling unit and a $3.69 \mu \mathrm{m}$ per pixel monochrome camera (Grasshopper3, FLIR Systems). The substrate holder assembly was supported by two closed-loop piezo-based stages (Attocube Systems AG), which provided precise vertical and horizontal motion. Vertical motion was used to position the substrate surface relative to the laser focal point.

Detection of thermionic emission and signal processing. Thermionic emission was detected using a programmable current amplifier (CA5350, NF Corporation) electrically connected to the metal substrate via a copper substrate holder, BNC type vacuum feedthrough and cabling. The thermionic signal collected is the total, spatially integrated current emitted from the surface. The copper substrate holder, and therefore the signal collection circuitry, was electrically isolated from the chamber, including the stage, using a nonconductive PTFE block (Accu-Glass Products, Inc.). The current preamplifier was operated at ground potential for all measurements, i.e., there was no potential difference between the substrate and the vacuum chamber. To reduce signal noise during data collection the cables used for signal collection were shielded and electrical shielding, including BNC cable shielding, connected to the vacuum chamber, which was at ground potential. The noise floor of the current preamplifier was $<10 \mathrm{pA}$ at $10^{9} \mathrm{VA}^{-1}$ gain (see Supplementary Fig. 6 for measurement of the noise floor of the current preamplifier). Investigations were undertaken to locate spurious sources of noise. Notably the noise floor was not affected by the laser or galvanometer scanning mirrors, but the mass flow controller and vacuum gauges had measurable influence if positioned in line of sight of the substrate. The signal measured by the current amplifier, laser power and laser scan position were recorded using a FPGA based data acquisition system operating at $\sim 526 \mathrm{kHz}$ (USB-7856, National Instruments). This allowed direct correlation of the thermionic signal and laser parameters to metallographic features. The current amplifier settings were optimized for collection under high vacuum (gain $10^{4} \mathrm{~V} \mathrm{~A}^{-1}$, low-pass filter rise time $0 \mu \mathrm{s}$, signal rise time $0.7 \mu \mathrm{s}$ ) and argon (gain $10^{9} \mathrm{~V} \mathrm{~A}^{-1}$, low-pass filter rise time $30 \mu$ s, signal rise time $30.41 \mu \mathrm{s}$ ) chamber environments. The signals collected by the FPGA were post processed by smoothing the data over 11 discrete data points $(\sim 48 \mathrm{kHz})$. FFT analysis was performed using the Origin Pro software package (version 9.6).

Material characterization. Ex situ optical imaging of scanned hatch patterns on the substrate surface was performed using a Keyence VR-3000 wide-area 3D measurement system or a Keyence VK-X100 laser scanning confocal microscope. Substrates were cross sectioned using a water cooled, low-speed diamond cutoff saw, and then mounted in epoxy. The substrates were then ground using $\mathrm{SiC}$ paper down to 1200 grit, and polished using 3 and $1 \mu \mathrm{m}$ polycrystalline diamond suspension. Final vibratory polishing was performed using $0.05 \mu \mathrm{m}$ colloidal silica.
The substrate was etched electrolytically using a $10 \%$ oxalic acid solution at $6 \mathrm{~V}$ and imaged with a Keyence VHX-6000 optical microscope.

Surface height data of the laser tracks were collected ex situ using a Keyence VK-X100 laser scanning confocal microscope. Surface roughness, referred to as $S a$ as a function thermionic signal was determined using the collected surface heigh data by applying the arithmetic mean roughness method to discrete $64 \times 64 \mu^{2}$ regions along the center line of the laser tracks. Correlation between the thermionic signal and position on the laser tracks was enabled by measurement of the galvanometer scanning mirror positions as a function of time during irradiation. Regions $650 \mu \mathrm{m}$ from the start, end or turn points of the laser tracks where excluded from the analysis to ensure only steady state scan conditions were analyzed. A region size of a $64 \times 64 \mu \mathrm{m}^{2}$ was selected for the $S a$ calculation to ensure the region was within the $91 \mu \mathrm{m}$ laser beam diameter. The two-dimensional kernel density plot was produced by the Origin Pro software package using a bivariate kernel density estimator bandwidth method.

Thermal simulations. Simulation of the surface temperature and melt pool response during a single laser turn point irradiation was performed using the ALE3D multi-physics software tool, which is described in detail elsewhere ${ }^{4,11,22}$. The simulation was performed using a $1000 \times 300 \times 200 \mu \mathrm{m}^{3}$ bare substrate using the material properties of stainless steel $316 \mathrm{~L}$. Powder was not included in the simulation as it significantly increases the physical complexity. Laser parameters of $200 \mathrm{~W}$ power, $1500 \mathrm{~mm} \mathrm{~s}^{-1}$ steady state scan speed, $100 \mu \mathrm{m}$ hatch spacing and $91 \mu \mathrm{m}$ D $4 \sigma$ beam diameter were used in the simulation. During the laser turn point the scan speed was reduced to $750 \mathrm{~mm} \mathrm{~s}^{-1}$ and the laser power remained constant (for laser scan path information see Supplementary Fig. 7). The higher scan speeds used in the simulation compared to experiments were necessary due to the exhaustive computational requirements. The thermionic emission profile was calculated by applying the Richardson-Dushman equation to the simulated surface temperature profile, using the typical $A_{G}$ value $\left(60 \mathrm{~A} \mathrm{~cm}^{-2} \mathrm{~K}^{-2}\right)$, which is half of the upper theoretical limit ${ }^{41}$ and the $W(4.34 \mathrm{eV})$ value for stainless steel $304^{21}$. The material specific constants $W$ and $A_{G}$ vary with temperature ${ }^{21}$ and physical state of the material ${ }^{37}$; however, the values were assumed constant for this calculation due to uncertainty in the values at these high temperatures.

The normalized enthalpy term was calculated using $\frac{A P}{\pi \rho C T_{\mathrm{m}} \sqrt{\mathrm{Dua}^{3}}}$, where $A$ is the absorptivity of the material (assumed to be 0.4 under all conditions), $P$ is the laser power, $\rho$ is the density $\left(7.950 \mathrm{~g} \mathrm{~cm}^{-3}\right), C$ is the specific heat capacity $\left(0.79 \mathrm{~J} \mathrm{~g}^{-1}\right.$ $\left.\mathrm{K}^{-1}\right), T_{m}$ is the melting temperature $(1658 \mathrm{~K}), D$ is thermal diffusivity of the molten material $\left(0.0538 \mathrm{~cm}^{2} \mathrm{~s}^{-1}\right), u$ is the laser scan speed and $a$ is the $\frac{1}{e}$ radius of the laser beam $(a=\sigma \sqrt{2})^{24}$.

\section{Data availability}

Data needed to evaluate the conclusions in the paper are present in the paper and/or the Supplementary Information. Additional experimental and simulation data related to this paper may be requested from the corresponding author.

\section{Code availability}

ALE3D code along with computing resources are available through the HighPerformance Computing Innovation Center at LLNL. All data generated using this code is available from the corresponding author on reasonable request.

Received: 14 July 2020; Accepted: 30 October 2020; Published online: 27 November 2020

\section{References}

1. Wohlers, T. T. et al. Wohlers Report 2019. 3D Printing and Additive Manufacturing State of the Industry (Wohlers Associates, Fort Collins, 2019).

2. Grasso, M. \& Colosimo, B. M. Process defects and in situ monitoring methods in metal powder bed fusion: a review. Meas. Sci. Technol. 28, 044005 (2017).

3. Mehrpouya, M. et al. The potential of additive manufacturing in the smart factory industrial 4.0: a review. Appl. Sci. 9, 3865 (2019).

4. Khairallah, S. A. et al. Controlling interdependent meso-nanosecond dynamics and defect generation in metal 3D printing. Science 368, 660-665 (2020).

5. Everton, S. K., Hirsch, M., Stravroulakis, P., Leach, R. K. \& Clare, A. T. Review of in-situ process monitoring and in-situ metrology for metal additive manufacturing. Mater. Des. 95, 431-445 (2016).

6. Lane, B., Moylan, S., Whitenton, E. P. \& Ma, L. Thermographic measurements of the commercial laser powder bed fusion process at NIST. Rapid Prototyp. J. 22, 778-787 (2016).

7. Craeghs, T., Clijsters, S., Kruth, J. P., Bechmann, F. \& Ebert, M. C. Detection of process failures in layerwise laser melting with optical process monitoring. Phys. Procedia 39, 753-759 (2012)

8. Islam, M., Purtonen, T., Piili, H., Salminen, A. \& Nyrhilä, O. Temperature profile and imaging analysis of laser additive manufacturing of stainless steel. Phys. Procedia 41, 835-842 (2013). 
9. Cunningham, R. et al. Keyhole threshold and morphology in laser melting revealed by ultrahigh-speed X-ray imaging. Science 363, 849-852 (2019).

10. Martin, A. A. et al. Ultrafast dynamics of laser-metal interactions in additive manufacturing alloys captured by in situ X-ray imaging. Mater. Today Adv. 1, 100002 (2019).

11. Martin, A. A. et al. Dynamics of pore formation during laser powder bed fusion additive manufacturing. Nat. Commun. 10, 1987 (2019).

12. Zhao, C. et al. Real-time monitoring of laser powder bed fusion process using high-speed X-ray imaging and diffraction. Sci. Rep. 7, 3602 (2017).

13. Matthews, M. J. et al. Denudation of metal powder layers in laser powder bed fusion processes. Acta. Mater. 114, 33-42 (2016).

14. Furumoto, T., Alkahari, M. R., Ueda, T., Aziz, M. S. A. \& Hosokawa, A. Monitoring of laser consolidation process of metal powder with high speed video camera. Phys. Procedia 39, 760-766 (2012).

15. Stutzman, C. B., Nassar, A. R. \& Reutzel, E. W. Multi-sensor investigations of optical emissions and their relations to directed energy deposition processes and quality. Addit. Manuf. 21, 333-339 (2018).

16. Krauss, H., Eschey, C. \& Zaeh, M. Thermography for Monitoring the Selective Laser Melting Process. in Proc. 23rd Annual International Solid Freeform Fabrication Symposium 999-1014 (Laboratory for Freeform Fabrication and University of Texas at Austin, 2012).

17. DePond, P. J. et al. In situ measurements of layer roughness during laser powder bed fusion additive manufacturing using low coherence scanning interferometry. Mater. Des. 154, 347-359 (2018).

18. Kube, C. M., Shu, Y., Lew, A. J. \& Galles, D. Real-time characterization of laser-generated melt pools using ultrasound. Mater. Eval. 76, 525-534 (2018).

19. Wasmer, K., Kenel, C., Leinenbach, C. \& Shevchik, S. A. in Industrializing Additive Manufacturing-Proceedings of Additive Manufacturing in Products and Applications-AMPA2017 (eds. Meboldt, M. \& Klahn, C.) 200-209 (Springer International Publishing, 2018).

20. Herring, C. \& Nichols, M. H. Thermionic emission. Rev. Mod. Phys. 21, 185-270 (1949).

21. Wilson, R. G. Vacuum thermionic work functions of polycrystalline $\mathrm{Be}, \mathrm{Ti}, \mathrm{Cr}$, Fe, Ni, Cu, Pt, and type 304 stainless steel. J. Appl. Phys. 37, 2261-2267 (1966).

22. Khairallah, S. A., Anderson, A. T., Rubenchik, A. \& King, W. E. Laser powderbed fusion additive manufacturing: physics of complex melt flow and formation mechanisms of pores, spatter, and denudation zones. Acta. Mater. 108, 36-45 (2016).

23. Calta, N. P. et al. An instrument for in situ time-resolved X-ray imaging and diffraction of laser powder bed fusion additive manufacturing processes. Rev. Sci. Instrum. 89, 055101 (2018).

24. King, W. E. et al. Observation of keyhole-mode laser melting in laser powderbed fusion additive manufacturing. J. Mater. Process Technol. 214, 2915-2925 (2014).

25. Rubenchik, A. M., King, W. E. \& Wu, S. S. Scaling laws for the additive manufacturing. J. Mater. Process Technol. 257, 234-243 (2018).

26. Metelkova, J. et al. On the influence of laser defocusing in Selective Laser Melting of 316L. Addit. Manuf. 23, 161-169 (2018).

27. Hagelaar, G. J. M. \& Pitchford, L. C. Solving the Boltzmann equation to obtain electron transport coefficients and rate coefficients for fluid models. Plasma Sources Sci. Technol 14, 722 (2005).

28. Lieberman, M. A. \& Lichtenberg A. J. in Principles of Plasma Discharges and Materials Processing. 165-206 (John Wiley \& Sons, Inc., Hoboken, 2005).

29. Bidare, P., Bitharas, I., Ward, R. M., Attallah, M. M. \& Moore, A. J. Laser powder bed fusion at sub-atmospheric pressures. Int. J. Mach. Tools Manuf. 130-131, 65-72 (2018).

30. Calta, N. P. et al. Pressure dependence of the laser-metal interaction under laser powder bed fusion conditions probed by in situ X-ray imaging. Addit. Manuf. 32, 101084 (2020).

31. Sun, Z., Tan, X., Tor, S. B. \& Yeong, W. Y. Selective laser melting of stainless steel 316L with low porosity and high build rates. Mater. Des. 104, 197-204 (2016).

32. Ahmadi, A. et al. Effect of manufacturing parameters on mechanical properties of 316L stainless steel parts fabricated by selective laser melting: a computational framework. Mater. Des. 112, 328-338 (2016).

33. Baeva, M., Loffhagen, D., Becker, M. M. \& Uhrlandt, D. Fluid modelling of DC argon microplasmas: effects of the electron transport description. Plasma Chem. Plasma Process 39, 949-968 (2019).

34. Trapp, J., Rubenchik, A. M., Guss, G. \& Matthews, M. J. In situ absorptivity measurements of metallic powders during laser powder-bed fusion additive manufacturing. Appl. Mater. Today 9, 341-349 (2017).

35. Smith, J. R., Bilbro, G. L. \& Nemanich, R. J. Theory of space charge limited regime of thermionic energy converter with negative electron affinity emitter.
J. Vac. Sci. Technol. B Microelectron. Nanometer Struct. Process. Meas. Phenom. 27, 1132-1141 (2009).

36. Cunningham, R., Narra, S. P., Montgomery, C., Beuth, J. \& Rollett, A. D. Synchrotron-based X-ray microtomography characterization of the effect of processing variables on porosity formation in laser power-bed additive manufacturing of Ti-6Al-4V. JOM 69, 479-484 (2017).

37. Dushman, S. Thermionic emission. Rev. Mod. Phys. 2, 381-476 (1930).

38. Nassar, A. R. et al. Formation processes for large ejecta and interactions with melt pool formation in powder bed fusion additive manufacturing. Sci. Rep. $\mathbf{9}$, $1-11$ (2019)

39. Ly, S., Rubenchik, A. M., Khairallah, S. A., Guss, G. \& Matthews, M. J. Metal vapor micro-jet controls material redistribution in laser powder bed fusion additive manufacturing. Sci. Rep. 7, 4085 (2017).

40. Greiner, M. T., Chai, L., Helander, M. G., Tang, W.-M. \& Lu, Z.-H. Transition metal oxide work functions: the influence of cation oxidation state and oxygen vacancies. Adv. Funct. Mater. 22, 4557-4568 (2012).

41. Reimann, A. L. The apparent thermionic constant A of clean metals. Nature 133, 833-833 (1934).

\section{Acknowledgements}

This work was performed under the auspices of the U.S. Department of Energy (DOE) by Lawrence Livermore National Laboratory (LLNL) under Contract No. DEAC52-07NA27344. Project 19-FS-037 (experimental) and 18-SI-003 (simulation) were funded by the LDRD Program at LLNL. We would like to thank E.V. Garza and D.L. Rosas for cross sectioning and imaging of the keyhole transition substrate.

\section{Author contributions}

P.J.D. and A.A.M. conceived the idea. P.J.D., J.C.F., and A.A.M. conducted the experiments and analyzed the data with help from J.R.A., G.G., and M.J.M. S.A.K. conducted the computer simulations and analyzed the results with help from P.J.D., M.J.M., and A.A.M. P.J.D., M.J.M., and A.A.M. wrote the paper with input from all authors.

\section{Competing interests}

P.J.D. and A.A.M. are inventors of a patent, which describes thermionic emission detection during LPBF additive manufacturing and is assigned to LLNL. A.A.M and P.J. D. Laser powder bed fusion additive manufacturing in-process monitoring and optimization using thermionic emission detection. International publication number WO 2020/005228, World Intellectual Property Organization, International publication date 2 January 2020. All other authors declare no competing interests.

\section{Additional information}

Supplementary information is available for this paper at https://doi.org/10.1038/s43246 020-00094-y.

Correspondence and requests for materials should be addressed to A.A.M.

Peer review information Primary handling editor: John Plummer

Reprints and permission information is available at http://www.nature.com/reprints

Publisher's note Springer Nature remains neutral with regard to jurisdictional claims in published maps and institutional affiliations.

\section{(i)}

Open Access This article is licensed under a Creative Commons Attribution 4.0 International License, which permits use, sharing, adaptation, distribution and reproduction in any medium or format, as long as you give appropriate credit to the original author(s) and the source, provide a link to the Creative Commons license, and indicate if changes were made. The images or other third party material in this article are included in the article's Creative Commons license, unless indicated otherwise in a credit line to the material. If material is not included in the article's Creative Commons license and your intended use is not permitted by statutory regulation or exceeds the permitted use, you will need to obtain permission directly from the copyright holder. To view a copy of this license, visit http://creativecommons.org/ licenses/by/4.0/

(c) The Author(s) 2020 